\title{
Diabetes in the Emergency Department and Hospital: Acute Care of Diabetes Patients
}

\author{
William Ford • Wesley H. Self • Corey Slovis • \\ Candace D. McNaughton
}

Published online: 13 February 2013

(c) Springer Science+Business Media New York 2013

\begin{abstract}
Diabetes is a common condition, afflicting more than $20 \%$ of the American population over the age of 60 years. Patients with diabetes, particularly those with lower socioeconomic status or limited access to primary care, frequently seek care in hospital emergency departments. Emergency medicine physicians and hospitalists should coordinate care to improve glycemic control while maintaining safety. This may help to overcome clinical inertia and improve long term clinical outcomes for patients who seek acute medical care for diabetes.
\end{abstract}

Keywords Diabetes - Diabetic ketoacidosis .

Hyperglycemic hyperosmolar state $\cdot$ Emergency $\cdot$ Hospital

\section{Emergency Department Evaluation of Hyperglycemia and Hypoglycmia}

\section{Hyperglycemic Crisis: DKA and HHS}

Diabetic ketoacidosis (DKA) accounts for more than 110,000 hospitalizations annually in the United States, with mortality ranging from 2 to $10 \%$ [1-3]. Hyperglycemic hyperosmolar state (HHS) is much less common but confers a much greater mortality [4]. Patients with DKA classically present with uncontrolled hyperglycemia, metabolic acidosis, and increased total body ketone concentration. On the

\section{W. Ford}

Department of Medicine, Temple University, School of

Medicine, Philadelphia, PA, USA

W. H. Self · C. Slovis · C. D. McNaughton ( $\square)$

Department of Emergency Medicine, Vanderbilt University

Medical Center, Nashville, TN, USA

e-mail: candace.mcnaughton@Vanderbilt.Edu other hand, HHS is defined by altered mental status caused by hyperosmolality, profound dehydration, and severe hyperglycemia without significant ketoacidosis [3, 5].

\section{Initial Evaluation}

In the emergency department, the primary goals are rapid evaluation and stabilization. All patients with severe hyperglycemia should immediately undergo assessment and stabilization of their airway and hemodynamic status, with consideration of administration of naloxone for all patients with altered mentation to reverse potential opiate overdose, and thiamine for all patients at risk for Wernicke's encephalopathy. In cases requiring intubation, the paralytic succinylcholine should not be used if hyperkalemia is suspected as it may acutely further elevate potassium. Immediate assessment should also include placing patients on oxygen, measure $\mathrm{O}_{2}$ saturation and cardiac monitoring as well as obtaining vital signs, a fingerstick glucose, intravenous (IV) access, and a 12-lead electrocardiogram to evaluate for arrhythmias and signs of hyper- and hypokalemia.

Emergency department evaluation should include a thorough clinical history and physical examination, as well as a venous blood gas [6, 7], complete blood count, basic metabolic panel, and urinalysis; a urine pregnancy test must be sent for all women with childbearing potential. An important goal of this evaluation is identification of a precipitating cause, particularly a reversible cause. The differential diagnosis for hyperglycemic crisis includes the "Five I's": infection, infarction, infant (pregnancy), indiscretion (including cocaine use, and insulin lack (nonadherence or inappropriate dosing).

Critically ill patients should undergo additional testing as clinically indicated, including a complete metabolic panel, serum osmolality, lactate, phosphate levels in 
malnourished patients, and cardiac markers for older patients or any adult patient in DKA who has chest pain or signs of heart failure. A urine drug screen, blood alcohol level, and aspirin and acetaminophen levels should be sent for any patient with unexplained DKA and all patients with HHS. Evaluation for infection or injury should be guided by history and the physical examination. Effective serum osmolality should be calculated. The corrected serum sodium is estimated by decreasing measured serum sodium by $1.6 \mathrm{mEq} / \mathrm{l}$ for every $100 \mathrm{mg} / \mathrm{dl}$ increase in blood glucose over a baseline of $100 \mathrm{mg} / \mathrm{dl}[8,9]$; for every $100 \mathrm{mg} /$ $\mathrm{dl}$ increment increase in blood glucose over $400 \mathrm{mg} / \mathrm{dl}$, decrease measured serum sodium by an additional $4 \mathrm{mEq} / \mathrm{l}$ [10].

While the evaluation and differential diagnoses for DKA and HHS are similar, they are distinct clinical entities that require different, nuanced therapies. Patients with DKA require both judicious volume resuscitation and insulin to reverse their ketoacidosis. Patients with HHS require fluid resuscitation first and foremost; they may or may not require insulin, and those who do rarely require a continuous infusion. An algorithm for the initial evaluation and treatment of patients with DKA can be found in Fig. 1 [11].

\section{Insulin Therapy}

For patients with DKA, insulin should not be administered until hypovolemia has been addressed and serum potassium is known to be greater than $3.5 \mathrm{mEq} / \mathrm{l}$. Giving insulin to patients with a serum potassium level less than $3.5 \mathrm{mEq} / \mathrm{l}$ may cause rapid intracellular shifting of potassium and precipitate life-threatening arrhythmias. Critically ill patients may be given insulin, with a loading dose of regular insulin $(0.1$ units $/ \mathrm{kg}$ body weight to a maximum of 10 units), followed by an infusion of regular insulin ( 0.1 units $/ \mathrm{kg}$ body weight $/ \mathrm{h}$, to a maximum of $10 \mathrm{units} / \mathrm{h}$ ) $[12,13]$. Less seriously ill patients with DKA should be started on an infusion of regular insulin at 0.1 units $/ \mathrm{kg}$ body weight/h without a loading dose to minimize the risk of hypoglycemia. Other doses and routes of insulin administration have been studied [14-16], but these have not been evaluated outside of the research setting or in critically ill patients [12].

Expert opinion regarding the use of insulin for patients with HHS is mixed [12, 13, 17]. Because some patients with HHS achieve euglycemia with fluid resuscitation alone [18], and given the theoretical risks of precipitating oliguric renal failure or cerebral edema in inadequately fluid-resuscitated patients [17], insulin should not be given as part of initial therapy $[19,20]$. However, if the patient's serum glucose does not decrease by $50-70 \mathrm{mg} / \mathrm{dl} / \mathrm{h}$ despite appropriate fluid management, a bolus of regular insulin intravenously at 0.1 units $/ \mathrm{kg}$ body weight to a maximum of 10 units may be given.

\section{Electrolyte Replacement}

Patients with DKA or HHS experience significant shifts in potassium during resuscitation that may trigger lifethreatening arrhythmias. Death during initial resuscitation of patients with DKA is usually caused by hyperkalemia, whereas hypokalemia is the most common cause of death after treatment has been initiated. Therefore, serum potassium should be checked every $2 \mathrm{~h}$ until it has stabilized in all patients with hyperglycemic crisis, and these patients should remain on a cardiac monitor until they are back to baseline. Guidelines for the treatment of hyperkalemia and hypokalemia in patients with hyperglycemic crisis based on expert opinion and our clinical experience are found in Table 1 [11].

Serum phosphate levels are not routinely measured or followed in DKA but should be closely monitored at the time of presentation and during resuscitation for malnourished patients with DKA and HHS and in those patients who appear to have decreased respiratory drive. Bicarbonate infusion should not be used routinely in the treatment of DKA or HSS [13]. Expert opinion suggests that it may be used selectively in patients with DKA and severe acidosis ( $\mathrm{pH}$ less than 6.9) or in patients with severe hyperglycemia who present with a wide-complex or disorganized cardiac rhythm thought to be caused by hyperkalemia. Bicarbonate must always be used with care as it may prolong hypokalemia and hospitalization and cause paradoxical cerebral acidosis and even cerebral edema in children [17, 21-23].

\section{Stabilization and Disposition for Patients with DKA and HHS}

All patients with hyperglycemic crisis require finger-stick glucose measurements hourly until they have stabilized. For patients with DKA, an additional bolus of regular insulin of 0.1 units $/ \mathrm{kg}$ by IV should be given if the blood glucose level does not decrease by $10 \%$ in the first hour; the continuous infusion of regular insulin should then resume at the original dose. Dextrose should be added to the continuous IV fluids once blood glucose has decreased to less than $250 \mathrm{mg} / \mathrm{dl}$. Patients with HHS whose blood glucose level decreases to less than $300 \mathrm{mg} / \mathrm{dl}$ should be observed closely for signs of oliguric renal failure and cardiovascular collapse; these patients may require the addition of dextrose to IV fluids, even without administration of insulin. 
Fig. 1 Initial evaluation and treatment of DKA in the emergency department [11]. *Laboratory studies: CBC, BMP, VBG, UA, urine pregnancy test if female, childbearing age; if critically ill or clinically indicated: CMP, serum osmolality, phosphate, lactate, cardiac markers, urine drug screen, blood alcohol level, chest X-ray, or other imaging studies; calculate: effective serum osmolality, corrected serum sodium. ${ }^{\text {TS}}$ Signs of critical illness include 1 altered mental status, 2 signs of hypoperfusion, 3 significant derangement in $\mathrm{HR}, \mathrm{BP}, \mathrm{RR}$, temperature, or $\mathrm{O}_{2}$ sat, or 4 signs of severe acidosis such as marked Kussmaul respirations. $C B C$ complete blood count; $B M P$ basic metabolic panel; $V B G$ venous blood gas; $U A$ urinalysis; $C M P$ complete metabolic panel; $\mathrm{O}_{2}$ oxygen; $E C G$

electrocardiogram; $B P$ blood pressure; $H R$ heart rate; $R R$ respiratory rate; temp temperature; $N S$ normal saline; pt patient; IVP IV push. Copyright, Department of Emergency Medicine, Vanderbilt University Medical Center

\section{Initial Evaluation and Treatment of DKA \\ in the ED}

1. Secure the airway, breathing, and circulation

2. Consider or give naloxone, thiamine

3. Obtain fingerstick blood glucose; place pt on $\mathrm{O}_{2}$, cardiac monitor; obtain IV access, ECG; measure $\mathrm{O}_{2}$ sat, $\mathrm{BP}, \mathrm{HR}, \mathrm{RR}$, temp

4. Obtain blood for laboratory studies, including VBG ${ }^{*}$

5. Initiate therapy based on acuity

Critically III ${ }^{\mp}$

IVF: NS bolus $1 \mathrm{~L}$, then $500 \mathrm{ml} / \mathrm{hr}$

Insulin, Regular: bolus $0.1 \mathrm{U} / \mathrm{kg}$, max 10U IVP; then continuous infusion $0.1 \mathrm{U} / \mathrm{kg} / \mathrm{hr}$

Potassium: See Table 3

*****

Phosphate: serum level $<1.5 \mathrm{mg} / \mathrm{dL}$, start $\mathrm{K}_{2} \mathrm{PO}_{4} 0.5 \mathrm{ml} / \mathrm{hr}$

Bicarbonate: ONLY if $\mathrm{pH}<6.9$ or wide, agonal rhythm due to hyperk

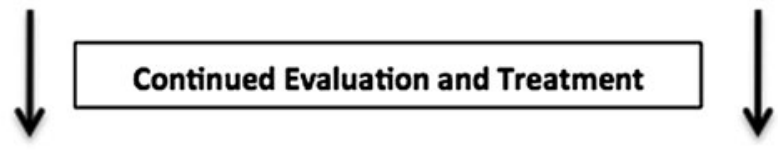

Fingerstick blood glucose q1hr VBG, BMP alternating $q 2 \mathrm{hr}$ Continuous cardiac monitor If blood glucose does not fall by $10 \%$ in the first hour, rebolus with insulin $0.14 \mathrm{U} / \mathrm{kg}$ IV, then continue previous continuous infusion
Not Critically III

IVF: based on pt's fluid status Insulin, Regular: +/- bolus; continuous infusion of $0.1 \mathrm{U} / \mathrm{kg} / \mathrm{hr}$, max 10U IVP

Potassium: See Table 3

****

Phosphate: N/A

Bicarbonate: N/A

Once serum glucose $<200 \mathrm{mg} / \mathrm{dL}$, reduce insulin infusion to $0.02-0.05 \mathrm{U} / \mathrm{kg} / \mathrm{hr}$

Maintain serum glucose between $150-200$ $\mathrm{mg} / \mathrm{dL}$ until resolution of DKA

To transition from IV to SC insulin, continue IV infusion 1-2 hours after $\mathrm{SC}$ insulin has been administered

Table 1 Replacement thresholds for potassium in hyperglycemic crisis [11]

\begin{tabular}{ll}
\hline Serum potassium $(\mathrm{mEq} / \mathrm{l})$ & Action \\
\hline$>5.3$ & No additional potassium; recheck in $1 \mathrm{~h}$ \\
$4.0-5.3$ & Add $\mathrm{KCl} 10 \mathrm{mEq} / \mathrm{l} / \mathrm{h}$ to IV fluids \\
$3.5-<4.0$ & Add $\mathrm{KCl} 20 \mathrm{mEq} / \mathrm{l} / \mathrm{h}$ \\
$<3.5$ & Hold insulin \\
& Add $\mathrm{KCl} 20-60 \mathrm{mEq} / \mathrm{l} / \mathrm{h}$ \\
& Continuous cardiac monitoring \\
\hline
\end{tabular}

Copyright, Department of Emergency Medicine, Vanderbilt University Medical Center
In the treatment of DKA, expert opinion recommends continuing an insulin infusion until acidosis has resolved and an anion gap is no longer present, but there are no formally endorsed criteria regarding when to discontinue insulin infusions. As a general rule, when converting to subcutaneously given insulin, a subcutaneous dose should be given $1 \mathrm{~h}$ before stopping the IV infusion to prevent rebound hyperglycemia [12, 13]. Each institution should devise a standard protocol to provide consistent care for its patients [24].

Patients with DKA or HHS rarely meet criteria to be safely discharged from emergency departments [25]. These 
patients typically require admission to the hospital with hourly blood glucose and neurological checks until they have stabilized.

\section{Hypoglycemia Emergencies}

In clinical practice, hypoglycemia is generally defined as a blood glucose level less than $60 \mathrm{mg} / \mathrm{dl}$ [26]. Severe hypoglycemia, requiring the assistance of another person to regain euglycemia, can cause significant morbidity, particularly in the chronically ill or elderly; it is the cause of death in approximately $3 \%$ of insulin-dependent diabetic patients [27]. Hypoglycemia is one of the most frequent diabetes-related chief complaints seen in emergency departments [28].

Oral-Hypoglycemic Agents and Insulin as a Cause of Hypoglycemia

While biguanides such as metformin, alpha-glucosidase inhibitors, and thiazolidinediones do not cause hypoglycemia when used in isolation, sulfonylureas and meglitinides increase insulin secretion and activity and therefore can cause hypoglycemia [29]. The half-life for most sulfonylurea medications is $14-16 \mathrm{~h}$; they can cause severe, prolonged hypoglycemia. Although meglitinides have a shorter half-life, the risk of recurrent hypoglycemia from these medications is unknown; experts urge caution and recommend assuming that these patients have a high risk of recurrent hypoglycemia [30]. Exogenous insulin administered in error or in attempt to self-harm should also be considered in the differential diagnosis of potential causes of hypoglycemia.

\section{Treatment of Hypoglycemia}

As with patients who present in hyperglycemic crisis, the initial evaluation of patients with suspected hypoglycemia includes securing the airway and assessing hemodynamic status. Altered mentation and signs of malnutrition should prompt administration of naloxone and consideration for intravenous thiamine, respectively. Naloxone and dextrose may completely reverse coma and eliminate the need for intubation; whenever possible, they should be given before proceeding to intubation.

Thiamine at $100 \mathrm{mg}$ IV should be administered to all patients who present with altered mentation and any signs of malnutrition or history suggestive of malabsorption, including prior gastric bypass surgery [31]. Despite traditional teaching to the contrary, there is no evidence that a dextrose load without thiamine replacement acutely precipitates Wernicke's encephalopathy. Treatment with dextrose should not be delayed if thiamine is not immediately available [32].

Once bedside glucose measurement confirms hypoglycemia, treatment should be accomplished immediately with either oral intake of food, dextrose given IV, or intramuscularly given glucagon. When decreased mentation precludes oral intake of food, dextrose given IV is the first-line therapy. One amp of dextrose provides approximately 100 calories, raising blood glucose levels for at least 20-30 min (Fig. 2 [11]). Glucagon $1 \mathrm{mg}$ may be administered subcutaneously or intramuscularly if IV access is not available, but it requires $15 \mathrm{~min}$ or more for onset of action and is associated with vomiting. The treatment for hypoglycemia may have a shorter duration of action than many of the precipitating drugs, and patients who are successfully treated initially may require repeat doses of glucose. Additionally, depending on the cause of hypoglycemia (e.g., sulfonylurea ingestion), administration of dextrose may precipitate rebound hypoglycemia.

Until blood glucose levels have clearly stabilized, they should be checked hourly at a minimum. Patients who develop recurrent hypoglycemia or whose blood glucose levels decrease despite administration of dextrose should be started on continuous IV dextrose with more frequent blood glucose level checks, and they should be hospitalized in the intensive care unit for continued evaluation and treatment.

\section{Disposition of Patients with Hypoglycemia}

In order to reasonably ensure no recurrence of hypoglycemia, all of the following criteria should be met prior to discharge of the patient from the emergency department [33]:

The episode of hypoglycemia was:

- Isolated (a single episode of hypoglycemia without recurrence)

- Completely and rapidly reversed without the need for a continuous dextrose infusion

- The result of an identified cause that is unlikely to cause recurrence

- Accidental

- Not caused by an oral hypoglycemic medication or long-acting insulin

And the patient:

- Has completed an uneventful 4-h observation period with serial blood glucose measurements in the normal range and not trending downward

- Has eaten a full meal during the observation period 
Fig. 2 Treatment of hypoglycemia [11]. Copyright, Department of Emergency

Medicine, Vanderbilt University

Medical Center
Initial Treatment

of Hypoglycemia

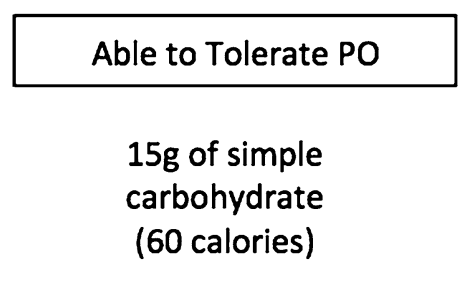

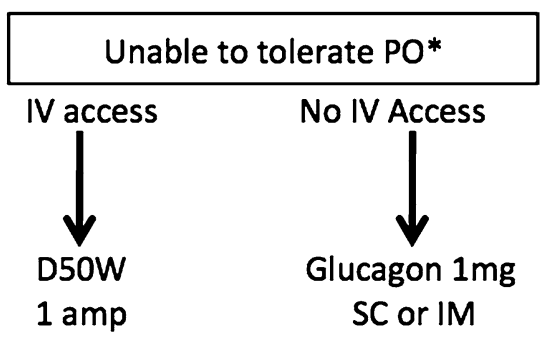

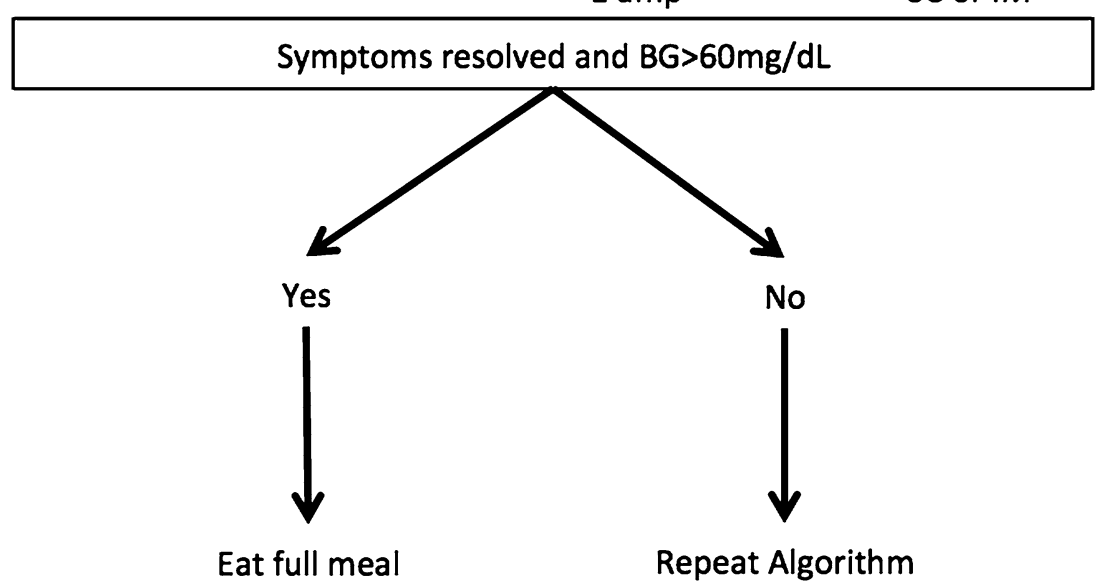

Eat full meal

Repeat Algorithm

\section{* Consider giving thiamine}

- Has no comorbid conditions that interfere with proper administration of medications and intake of food

- Understands how to prevent future episodes of hypoglycemia

- Can accurately monitor blood glucose at home

- Will be with a responsible adult who will monitor the patient

- Has close, reliable follow-up with a primary care provider

Expert opinion states that patients who meet these criteria and are discharged from the emergency department should reduce their insulin dose by $25 \%$ for at least the next $24 \mathrm{~h}$ to reduce the risk of recurrent hypoglycemia. Coordination with the patient's primary care provider is essential.

Many states require that patients with symptomatic hypoglycemia refrain from driving for 6-12 months and obtain clearance from a physician before resuming driving. Research has shown that this issue is rarely addressed in the emergency department [34]; for public health and the safety of patients, it must be included in discharge instructions, either at the time of discharge from the emergency department or from the hospital.

Patients who do not meet all of the above discharge criteria warrant hospital admission. Oral hypoglycemic agents and intermediate- or long-acting forms of insulin (lente, NPH, glargine, and ultralente) are likely to cause recurrent hypoglycemia, and patients taking these medications generally should be admitted. Patients with hypoglycemia that cannot be completely reversed, was caused by massive ingestion or overdose, or is associated with sepsis, starvation, liver failure, or adrenal insufficiency should be admitted to the hospital's intensive care unit. Suicide attempts, factious disorder, or other psychiatric illnesses should be addressed with a psychiatric consultation.

Patients with Undiagnosed Pre-diabetes or Diabetes

Of the 23.6 million Americans with diabetes in 2007, 5.7 million were unaware they had the disease. Additionally, 54 million Americans had pre-diabetes (impaired fasting glucose and impaired glucose tolerance) [25]. Patients who 
present to the emergency department are at high risk for undiagnosed diabetes [35]; in one study, nearly four out of five patients in an urban emergency department met American Diabetes Association (ADA) criteria for diabetes screening [36].

The diagnosis of diabetes requires testing performed on two separate occasions unless "unequivocal hyperglycemia" is present $[37,38 \bullet, 39]$. Patients with unequivocal signs of hyperglycemia such as polyuria, polydipsia, nocturia, or acidosis are likely to need hospital admission, where further testing may be performed. Using an insulin protocol, treatment of hyperglycemia should ideally begin before admission and should not be delayed by confirmatory testing [40]. This requires close and clear communication and collaboration between the emergency physician and the hospitalist.

Although hospital emergency departments cannot offer confirmatory testing, patients who are at high risk for prediabetes or diabetes can be identified, and these patients should be counseled regarding their likely diagnosis and lifestyle interventions. A prospective cohort study conducted in an urban emergency department found that all patients with risk factors for diabetes (age greater than 45 years, polyuria, and polydipsia) and a random blood glucose greater than $155 \mathrm{mg} / \mathrm{dl}$ were later diagnosed with pre-diabetes or diabetes [40]. The use of $\mathrm{A}_{1} \mathrm{C}$ as a screening tool in emergency departments is being studied [36] but is not currently recommended [39]. For patients who are hospitalized, communication with the Hospitalist physicians regarding patients at high risk for undiagnosed or uncontrolled diabetes may help to overcome clinical inertia and improve glycemic control.

Particularly for patients who are discharged from the emergency department, it is imperative that patients with suspected pre-diabetes or diabetes be made aware of their abnormal test results and the need for urgent follow-up with a primary care provider. These patients should be counseled about lifestyle changes. For patients who are hospitalized, clear communication with the Hospitalist team regarding evaluation completed in the emergency department and potential initiation of therapeutics after hospitalization may improve glycemic control for hospitalized patients.

\section{Glycemic Control After Hospitalization}

There is vast discrepancy among hospitalists regarding glycemic management goals throughout the US. This is despite data supporting improved patient outcomes and hospital stays [41]. Recently studies have suggested that "tight" glycemic control in the hospital setting may lead to an increase in mortality due to hypoglycemia [42].
However, the level of glycemic control in those studies was much "tighter" than recommended in the AACE/ADA guidelines for random blood glucose goals [41]. It is common for hospitalists to allow blood glucose levels to fluctuate during a patient's hospital stay. The traditional hospitalist training, primarily in an internal medicine residency program, often consists of treating diabetic patients with a regular insulin sliding scale. Diabetes management was not thought to be an issue of critical importance until blood sugars consistently were above $300 \mathrm{mg} / \mathrm{dl}$ or acidosis reared its head. The nature of hospital medicine is to be involved in all aspects patient care. Glycemic control should be viewed differently in lieu of data linking improved patient outcomes and eugylcemia [43].

The American Association of Clinical Endocrinologists and the American Diabetes Association published their revised consensus statement on the inpatient standards for glycemic control in 2009 [41]. Overall their guidelines had remained consistent with slight alterations in overall blood sugar levels. The following is a summary of those recommendations [41]:

- Critically ill patients

- Close monitoring for episodes of hypoglycemia

- Insulin therapy should be initiated for patients with blood glucose of $180 \mathrm{mg} / \mathrm{dl}$

- Goal blood sugars for ICU patients should be $140-180 \mathrm{mg} / \mathrm{dl}$

- Non-critically ill patients (medical/surgical floor patients

- Pre-meal (fasting) blood glucose goal of $<140 \mathrm{mg} / \mathrm{dl}$

- Random blood glucose levels of $<180 \mathrm{mg} / \mathrm{dl}$

- Sliding scale insulin is NOT the preferred method inpatient management

- Basal and meal time (prandial) insulin with correctional insulin are the preferred treatment regimens

- Non-insulin (oral) anti-hyperglycemics are NOT recommended while patients are hospitalized

Some physicians argue that the above treatment goals are too stringent and potentially can cause harm in the area of hypoglycemia [44]. This argument must continue to occur for patient safety, however allowing blood sugars to remain $>200 \mathrm{mg} / \mathrm{dl}$ is not an acceptable target. While hypoglycemia is a real risk of over correction, the medical community must realize that neglecting hyperglycemia can potentially be as harmful [45•].

Protocol driven medicine is beginning to confront the problem of hyperglycemia. As systems are now able to identify outliers and "remind" providers that basal in 
conjunction with meal time and correctional insulin are the preferred method, we are getting closer to our goal for inpatient management [46].

Physicians may ask, in addition to improved patient outcomes, why should one bother with a blood sugar when a patient will only be in the hospital for 3-4 days? The answer is quite simple: quality of care and patient outcomes. Currently, The Joint Commission has implemented tight glycemic control as one of its quality indicators for the Surgical Care Improvement Project [47]. This initiative began in 2006 and will have real financial implications for the hospital. As hospitalists engage in more surgical comanagement glycemic control will become more visible. In this area glycemic control has been equated with improved morbidity and a decrease in length of stay [48].

\section{Breakdown in Communication}

Glycemic control should begin on admission, preferably during the emergency department evaluation. This rings true for most of patient care but is extremely important when dealing with blood sugars. When looking at systems, particularly institutions that focus on healthcare delivery, communication is the fundamental base by which are breakdown or success hinges. As hospitalists, the initial communication begins with the emergency department. There is a plethora of data supporting that improvement in this area leads to an improved patient experience and outcome [49•]. Poor communication leads to poor handoffs and medical errors. The interaction between the hospitalist and the emergency department physician exemplifies this problem. The rapid work pace typical of the ED and hospital is a barrier to clear communication and effective handoffs. The information exchanged is often minimal at best [48]. One area that needs increased focus during the $\mathrm{ED} /$ hospitalist exchange is glycemic control. ED and hospitalist physicians represent geographically based specialties and have an obligation to improve this hospital process. By including glycemic control in the conversation during patient admission, outcomes and performance measures are expected to improve. Clear expectations and goals regarding this communication must be set to create a good foundation between these two departments. All too often, ambiguous communication occurs between physicians and the information is delivered one way [48], often with the ED physician providing the majority of the information. Hospitalists may improve communication by inquiring about available new information, including glycemic levels and strategies. Standardization of the communication process may help to streamline this process. This may be accomplished through interdepartmental collaboration and goal achievement. Department and section heads must meet routinely and examine best practices and disseminate these best practices among all staff within their respective departments. Routine analysis and examination of glycemic control and adverse events such as hypoglycemic events or development of DKA after hospitalization may help to ensure that communication is occurring and the standardized procedures are being implemented as planned. Tight glycemic control is a standard that should be accepted and strived for in all hospitals settings $[41,45]$.

In order to have a successful communication exchange between the ED physician and the hospitalist, certain expectations must be met. First, clear expectation regarding data exchange must exist. This process must begin at the administrative level. Before improvement is initiated, goals must be agreed upon and conveyed to all engaged in patient care. Consensus regarding these goals and how best to achieve them should be the responsibility of onsite leadership. Referring to national glycemic standards should serve as a platform for those goals.

\section{Summary}

Patients with hypo- and hyperglycemia caused by diabetes frequently seek care in the emergency department and hospital. Emergency physicians must be skilled at diagnosing and stabilizing patients with hyper- and hypoglycemia. Emergency physicians and hospitalists should use protocols and clear communication to improve acute and chronic glycemic control for these patients.

Acknowledgments Dr. McNaughton is supported by the Vanderbilt Emergency Medicine Research Training (VEMRT) Program (HL 1K12HL109019). Dr. Self is supported by a KL2 grant from the National Institutes of Health and administered through the Vanderbilt Clinical and Translational Science Award (KL2 TR000446).

\section{References}

Papers of particular interest, published recently, have been highlighted as:

- Of importance

1. Wetterhall SF, Olson DR, DeStefano F, et al. Trends in diabetes and diabetic complications, 1980-1987. Diabetes Care. 1992;15: 960-7.

2. Lebovitz HE. Diabetic ketoacidosis. Lancet. 1995;345:767-72.

3. Bagdade JD. Endocrine emergencies. Med Clin North Am. 1986;70:1111-28.

4. Lorber D. Nonketotic hypertonicity in diabetes mellitus. Med Clin North Am. 1995;79:39-52.

5. Atchley DW, Loeb RF, Richards DW, Benedict EM, Driscoll ME. On diabetic acidosis: a detailed study of electrolyte balances following the withdrawal and reestablishment of insulin therapy. J Clin Investig. 1933;12:297-326. 
6. Brandenburg MA, Dire DJ. Comparison of arterial and venous blood gas values in the initial emergency department evaluation of patients with diabetic ketoacidosis. Ann Emerg Med. 1998;31: 459-65.

7. Ma OJ, Rush MD, Godfrey MM, Gaddis G. Arterial blood gas results rarely influence emergency physician management of patients with suspected diabetic ketoacidosis. Acad Emerg Med. 2003;10:836-41.

8. Katz MA. Hyperglycemia-induced hyponatremia: calculation of expected serum sodium depression. New Engl J Med. 1973;289: 843-4.

9. Crandall ED. Letter: serum sodium response to hyperglycemia. New Engl J Med. 1974;290:465.

10. Hillier TA, Abbott RD, Barrett EJ. Hyponatremia: evaluating the correction factor for hyperglycemia. Am J Med. 1999;106: 399-403.

11. McNaughton CD, Self WH, Slovis C. Diabetes in the emergency department: acute care of diabetes patients. Clin Diabetes. 2011; 29:51-9.

12. Kitabchi AE, Umpierrez GE, Miles JM, Fisher JN. Hyperglycemic crises in adult patients with diabetes. Diabetes Care. 2009;32: 1335-43.

13. Magee MF, Bhatt BA. Management of decompensated diabetes. Diabetic ketoacidosis and hyperglycemic hyperosmolar syndrome. Crit Care Clin. 2001;17:75-106.

14. Kitabchi AE, Murphy MB, Spencer J, Matteri R, Karas J. Is a priming dose of insulin necessary in a low-dose insulin protocol for the treatment of diabetic ketoacidosis? Diabetes Care. 2008; 31:2081-85.

15. Kitabchi AE, Umpierrez GE, Fisher JN, Murphy MB, Stentz FB. Thirty years of personal experience in hyperglycemic crises: diabetic ketoacidosis and hyperglycemic hyperosmolar state. J Clin Endocrin Metab. 2008;93:1541-52.

16. Fisher JN, Shahshahani MN, Kitabchi AE. Diabetic ketoacidosis: low-dose insulin therapy by various routes. New Engl J Med. 1977;297:238-41.

17. Filbin MR, Brown DF, Nadel ES. Hyperglycemic hyperosmolar nonketotic coma. J Emerg Med. 2001;20:285-90.

18. West ML, Marsden PA, Singer GG, Halperin ML. Quantitative analysis of glucose loss during acute therapy for hyperglycemic hyperosmolar syndrome. Diabetes Care. 1986;9:465-71.

19. Carroll P, Matz R. Uncontrolled diabetes mellitus in adults: experience in treating diabetic ketoacidosis and hyperosmolar nonketotic coma with low-dose insulin and a uniform treatment regimen. Diabetes Care. 1983;6:579-85.

20. Matz R. Management of the hyperosmolar hyperglycemic syndrome. Am Fam Phys. 1999;60:1468-76.

21. Morris LR, Murphy MB, Kitabchi AE. Bicarbonate therapy in severe diabetic ketoacidosis. Ann Intern Med. 1986;105:836-40.

22. Viallon A, Zeni F, Lafond P, et al. Does bicarbonate therapy improve the management of severe diabetic ketoacidosis? Crit Care Med. 1999;27:2690-93.

23. Green SM, Rothrock SG, Ho JD, et al. Failure of adjunctive bicarbonate to improve outcome in severe pediatric diabetic ketoacidosis. Ann Emerg Med. 1998;31:41-8.

24. Kanji S, Singh A, Tierney M, Meggison H, McIntyre L, Hebert PC. Standardization of intravenous insulin therapy improves the efficiency and safety of blood glucose control in critically ill adults. Intensive Care Med. 2004;30:804-10.

25. CDC. 2011 National diabetes fact sheet. Atlanta: Centers for Disease Control and Prevention, 2007. http://www.cdc.gov/ diabetes/pubs/factsheets.htm. Accessed 7 Feb 2011.

26. Osorio I, Arafah BM, Mayor C, Troster AI. Plasma glucose alone does not predict neurologic dysfunction in hypoglycemic nondiabetic subjects. Ann Emerg Med. 1999;33:291-8.
27. The Diabetes Control and Complications Trial Research Group. Hypoglycemia in the diabetes control and complications trial. Diabetes. 1997;46:271-86.

28. Brackenridge A, Wallbank H, Lawrenson RA, Russell-Jones D. Emergency management of diabetes and hypoglycaemia. Emerg Med J. 2006;23:183-5.

29. Harrigan RA, Nathan MS, Beattie P. Oral agents for the treatment of type 2 diabetes mellitus: pharmacology, toxicity, and treatment. Ann Emerg Med. 2001;38:68-78.

30. Rowden AK, Fasano CJ. Emergency management of oral hypoglycemic drug toxicity. Emerg Med Clin North Am. 2007;25: 347-56. (abstract viii).

31. Donnino MW, Vega J, Miller J, Walsh M. Myths and misconceptions of Wernicke's encephalopathy: what every emergency physician should know. Ann Emerg Med. 2007;50:715-21.

32. Hack JB, Hoffman RS. Thiamine before glucose to prevent Wernicke encephalopathy: examining the conventional wisdom. J Am Med Assoc. 1998;279:583-4.

33. Adams J. Emergency medicine. 2nd ed. Philadelphia: Saunders Elsevier; 2014.

34. Ginde AA, Savaser DJ, Camargo CA Jr. Limited communication and management of emergency department hyperglycemia in hospitalized patients. J Hosp Med. 2009;4:45-9.

35. Ginde AA, Delaney KE, Lieberman RM, Vanderweil SG, Camargo CA Jr. Estimated risk for undiagnosed diabetes in the emergency department: a multicenter survey. Acad Emerg Med. 2007; 14:492-5.

36. Ginde AA, Cagliero E, Nathan DM, Camargo CA Jr. Value of risk stratification to increase the predictive validity of $\mathrm{HbA} 1 \mathrm{c}$ in screening for undiagnosed diabetes in the US population. J Gen Intern Med. 2008;23:1346-53.

37. American Diabetes Association. Standards of medical care in diabetes 2008. Diabetes Care. 2008;31(Suppl 1):S12-54.

38. - Executive summary: standards of medical care in diabetes, 2011. Diabetes Care. 2011;34(Suppl 1):S4-10. This paper summarizes the recommendations regarding care for patients with diabetes and provides the most recent methods for defining diabetes.

39. American Diabetes Association. Standards of medical care in diabetes, 2011. Diabetes Care. 2011;34(Suppl 1):S11-61.

40. Charfen MA, Ipp E, Kaji AH, Saleh T, Qazi MF, Lewis RJ. Detection of undiagnosed diabetes and prediabetic states in highrisk emergency department patients. Acad Emerg Med. 2009;16: 394-402.

41. Moghissi ES, Korytkowski MT, DiNardo M, et al. American association of clinical endocrinologists and American diabetes association consensus statement on inpatient glycemic control. Diabetes Care. 2009;32:1119-31.

42. Finfer S, Chittock DR, Su SY, et al. Intensive versus conventional glucose control in critically ill patients. New Engl J Med. 2009;360:1283-97.

43. Griesdale DE, de Souza RJ, van Dam RM, et al. Intensive insulin therapy and mortality among critically ill patients: a meta-analysis including NICE-SUGAR study data. CMAJ Canad Med Assoc J. 2009;180:821-7.

44. Inzucchi SE, Siegel MD. Glucose control in the ICU: how tight is too tight? New Engl J Med. 2009;360:1346-49.

45. - Umpierrez GE, Hellman R, Korytkowski MT, et al. Management of hyperglycemia in hospitalized patients in non-critical care setting: an endocrine society clinical practice guideline. J Clin Endocrinol Metab. 2012;97:16-38. This paper reviews the most recent recommendations for recommendation for the treatment of patients with diabetes who are hospitalized.

46. Maynard G, Lee J, Phillips G, Fink E, Renvall M. Improved inpatient use of basal insulin, reduced hypoglycemia, and 
improved glycemic control: effect of structured subcutaneous insulin orders and an insulin management algorithm. J Hosp Med. 2009;4:3-15.

47. Joint Commission. Surgical care improvement project. Oakbrook Terrace: The Joint Commission; 2012. http://www.jointcommiss ion.org/surgical_care_improvement_project/. Accessed 15 March 2012.

48. Bratzler DW, Hunt DR. The surgical infection prevention and surgical care improvement projects: national initiatives to improve outcomes for patients having surgery. Clin Infect Dis. 2006;43:322-30.

49. - Apker J, Mallak LA, Gibson SC. Communicating in the "gray zone": perceptions about emergency physician hospitalist handoffs and patient safety. Acad Emerg Med. 2007;14:884-94. This paper shows the viewpoints of the multiple members in the handoff of care between the emergency department and hospital and addresses issues that should be considered during transitions of care. 